\title{
Isotopic constraints on the mode of terrestrial planet formation
}

\author{
CHRISTOPH BURKHARDT ${ }^{1}$, FRIDOLIN SPITZER ${ }^{1}$, \\ ALESSANDRO MORBIDELLI ${ }^{2}$, GERRIT BUDDE ${ }^{3}$, JAN \\ RENDER $^{1}$, THOMAS KRUIJER ${ }^{4}$ AND THORSTEN KLEINE ${ }^{1}$ \\ ${ }^{1}$ University of Münster, Institut für Planetologie \\ ${ }^{2}$ Observatoire de la Cote d'Azur \\ ${ }^{3}$ California Institute of Technology \\ ${ }^{4}$ Museum für Naturkunde Berlin \\ Presenting Author: burkhardt@uni-muenster.de
}

Two contrasting modes of rocky planet formation are known, and it is debated which one built the terrestrial planets of the solar system. The classical model states that the terrestrial planets formed over tens of millions of years —long after the gas disk dissipated — via collisions among planetary embryos, which themselves formed from local planetesimals [1]. Alternatively, it has been argued that the terrestrial planets may have largely formed within the $\sim 5$ Ma lifetime of the gaseous disk by efficiently accreting large amounts of sunward-drifting outer solar system dust and pebbles [2]. The two models differ in the amount of outer solar system material in the terrestrial planets, which, in principle, may be determined using nucleosynthetic isotope variations among meteorites and planets. Yet, using this approach both low and high amounts of outer solar system material in Earth and Mars have been inferred [3,4].

Here we resolve these issues and show that the disparate results are linked to the decade-old question of how samples from the meteorite record relate to the building materials of the terrestrial planets. Using novel isotope data and anomaly correlations in multi-elemental isotope space we show that Earth and Mars must have incorporated material from the innermost solar system that remained unsampled among meteorites. By characterizing the composition of this 'lost' material, we find that the distinct isotopic compositions of Earth and Mars reflect variable mixtures of this material with objects akin to meteorites from the inner solar system, whereas the contribution of outer solar system material is limited to a few percent by mass. This is consistent with the classical model of terrestrial planet formation by collisions among local, inner solar system objects, but not with an origin by pebble accretion. The low fraction of outer solar system material in the terrestrial planets likely reflects the efficiency of Jupiter in preventing dust-drift from beyond its orbit, and thus bears testimony to the specific pathway of rocky planet formation in the solar system.

References: [1] Wetherill (1994) GCA 58. [2] Levison et al. (2015) PNAS, 112. [3] Dauphas (2017) Nature, 541 [4] Schiller et al. (2018) Nature, 555. 